\title{
Dynamic-Distributed Load Balancing for Highly-Performance and Responsiveness Distributed-GIS (D-GIS)
}

\author{
Aissatou Diasse $^{1,2}$, Foroski Kone ${ }^{1,2}$ \\ ${ }^{1}$ Faculty of Information Engineering, China University of Geosciences, Wuhan, China \\ ${ }^{2}$ GIS Software and Application Research Center of Ministry of Education, Wuhan, China \\ E-mail: \{diasseastou, kone69f\}@yahoo.fr \\ Received January 10, 2011; revised February 2, 2011; accepted February 15, 2011
}

\begin{abstract}
In recent years, several results have been introduced to enhance distributed GIS performance. While much more efforts have focused on tile map and simple symbologies on dynamic map, load balancing GIS servers have not been addressed by the GIS community so far. This paper, therefore, proposed dynamic distributed load balancing for D-GIS in order to quickly render information to client interface by involving a set of GIS servers which process clients' requests depending of an algorithm. In the model, several concepts were introduced and defined: Virtual Server within physical machine which constitutes a setup environment for a single GIS server, Load Hash Table which contains information about virtual server's capacity, real-time load and other mandatory elements, Request Split Table which splits requests depending of the input area's Quantity of Information and stores request tasks composition for later reconstitution. At last we have Distributed Failover Callback Function Table level one (respectively level two) which determines whether or not the request had been successfully processed by the chosen virtual server (respectively physical machine). This table allows sending back the same request to another virtual server (respectively physical node). Two load handlers (primary and secondary) are defined in case of failure. Our Model achieves efficient load balancing by: providing efficient node selection; optimizing request routing; managing node failover; involving client's request partitioning and introducing method type decomposition. A simulation of the algorithm shows a low response time when performing GIS operations.
\end{abstract}

Keywords: Load Balancing, Distributed-GIS, Performance, Responsiveness

\section{Introduction}

\subsection{History}

A centralized GIS, which provides an environment for desktop or standalone application is traditionally use. Now with the growth of internet techniques, D-GIS which are based-on distributed system are being used much more in place of traditional GIS. These systems used tens or hundreds of computers connected by high speed networks and have many advantages over standalone computers $[1,6,7]$. They provide resource sharing as one of their major advantages, which bring better performance and reliability than any other traditional system in the same conditions [2]. Like distributed system,
D-GIS are composed of autonomous hosts that are connected through a computer network [3]. They aim sharing of data and computation resources and collaboration on large scale applications [4]. Thus, the architecture of D-GIS is different from of traditional GIS because the system is built on the open and distributed internet environment [5]. D-GIS has two advantages. Firstly, they are designed with tools and features that are common to webpage and secondly they allow the dissemination of geographic data to multitude of clients and provide, access and perform GIS functions in the web.

\subsection{Problems}

Even with these advantages, recent use of this system 
shows that there exist shortcomings that D-GIS communities are faced. One of the critical problems faced is that of serving resources for multitudes of clients which has as consequence the decrease of performance and responsiveness of the applications. These are due among other to ${ }^{(1)}$ islands of technology used, ${ }^{(2)}$ large among of data transfer over the internet, ${ }^{(3)}$ numerous remote calls to a single GIS server and numerous heterogeneous devices. However, since productivity and efficiency are the business forces of organizations, GIS providers must seek for ways to break down the cause of low performance.

Several methods have been used to enhance D-GIS performance. One of such methods is the use of new data organization known as Tile Map, to reduce the data displaying and retrieving time. Nevertheless, very few studies had been focus on the load balancing of D-GIS servers which is one of the most successful methods to enhance applications performance in distributed systems computing. For those GIS systems that integrate load balancing into their applications, static load balancing is used which is inappropriate for D-GIS where tasks requirements' capacity vary frequently.

\subsection{Contributions}

In this paper we investigated, studied and designed dynamic load balancing algorithm for distributed GIS. We discussed and illustrated the use of virtual servers and double load balancer handlers. The system failover is managed by the introduction of two tables at two system levels to overcome virtual servers and physical machines failover. Moreover a request split style and a methoddecomposition are proposed to further enhance the performance of GIS applications. By taking as prototype a dynamic load balancing algorithm in peer to peer system, we modify, adjust and add some innovative parameters and tables on the algorithm to present a dynamic load balancing for distributed GIS systems.

\subsection{Method}

Firstly we defined and described the proposed model for balancing users' requests into a GIS servers cluster in section 2. Secondly, we went deeply into the model description with the different parameters, components, assumptions, architecture and algorithms. Finally we proposed a prototype implementation model of our distributed GIS system with the different distributed tiers. The simulation showed that the model fairly optimized the performance and responsiveness of the implemented applications based on our D-GIS designed and provided high availability in a scalable manner to meet future needs.

\section{Overview}

For proposing an efficient load balancing model for distributed GIS systems, we investigated some issues for a better responsiveness of implemented application.

- How can we balance the user's request between the GIS servers in the cluster efficiently?

- How can we handle client information that must be kept across the multiple requests?

- Is it the load balancing of the users' processes composed of several GIS tasks?

- How do we process the load balance when a request is composing of different GIS tasks?

- Where do we run a new job in the cluster when there is equal load on the different nodes?

- Are the costs to process a request the same regardless of which nodes are involved?

- Is the transfer of users' incoming request costless? Can it be negligible?

To implement the model for a satisfaction of the above statements, we impose to the distributed layers within the system to be balanced using an appropriate algorithm. Each layer is composed of several servers called cluster servers. The load balancer allows client's access to the cluster servers using a common domain name with a single virtual IP address. The load-balancing algorithm intercepts the incoming HTTP traffic and directs it to one of the servers in the cluster.

After deep study and considerations, we thought that the algorithms must be implemented in two different levels to provide highly available performance and responsiveness. The two cluster servers are the GIS servers cluster and the GIS services cluster. The first cluster contains the GIS services which balance users' requests using the Network Load Balancing. This method is not covered in this paper. The second cluster contains the GIS servers which use Dynamic Distributed Load Balancing (DDLB) algorithm. The paper is about the load balancing into GIS servers cluster.

\section{Dynamic-Distributed Load Balancing Model on GIS Servers Cluster}

\subsection{Model Definition}

In this section, we define the model proposed to balance the GIS servers cluster. A dynamic algorithm is used to balance user's requests. The algorithm uses the concept of virtual servers [11-16] to host processes. The basic idea of the model is to divide the machines into several virtual servers, store their information into a well known table called Load Hash Table (LHT) which is frequently updated to restore the real time nodes load, server 
failover likelihood and so on. When client requests a process provided by the GIS server, the LHT is used by the algorithm to search the appropriate virtual server and then send the client request to that specific server. If request is a getMap() or getInfo-OnInputArea() functions, input area is split depending of the area quantity of information. The proposed algorithm uses the utilization of the node instead of its load because virtual servers might in real life have heterogeneous capacity. However their capacity is used to balance the first incoming client request.

\subsection{Model Description}

For the GIS server cluster we proposed a dynamic load balancing algorithm which used a LHT as match up system between the nodes of the cluster and their utilizing load at any given time. The algorithm balances the clients' requests to nodes in the cluster, which yields a load-balanced system when the LHT maps nodes efficiently into the corresponding weight-load. The LHT is a concept we introduce in our distributed GIS that follow the hash table paradigm (HT). The HT or hash map is a data structure that uses a hash function to efficiently map certain identifiers or keys to associated values [17]. Whenever a specific virtual server is chosen it is stored, together with the client request in a table call distributed failover callback function table (DFCFT). This table allows the system to manage failover. The DFCFT contains the request status field which allows the parent node to be aware of the successful process and send the request to another node. However before processing client request the split request function is used to split client request into simple tasks and store the different tasks into a Split Request Table (SRT) in order to reconstitute the original request and render appropriate response. The dynamic distributed load balancing algorithm is characterized by a set of criteria describe in the follow points.

- Scalability: The node can be increased and decrease as wanted. Therefore even with ten, thousands or millions of virtual nodes the system will work correctly.

- Semi-Distributed: Each physical node has a copy of the DCFT in its memory and a general real time update must be done to update the tables in each node.

- Centralization: The nodes are collectively taken as slave node with a central coordination call master node. System store global information in the load balancer, so any of the slave nodes cannot be a master node.

- Fault Tolerance: The system is reliable; in fact the LHT, even with failover, continuously maps spe- cific nodes with a Upper Loaded: Upper Loaded $=$ $\mathrm{C}+\&$ such $\&>0$

- Availability: Even when servers within the cluster fail, the remaining servers are performing normally and can take over the failing server's request using either physical node level or cluster node level.

- Affinity: The system can manage a user's requests, either to a specific server or any server, depending on whether session information is maintained on the server or at an underlying database level.

- Adaptive: the algorithm is adapted to each incoming request due to the division of input area based on its quantity of Information.

- Bi-Load Balancers: The system is composed of two load balancers. For high-availability we proposed a primary load balancing and a secondary in case the load balancer fails.

\subsection{Model Composition}

\subsubsection{Virtual Server (VS)}

By using a known dynamic load balancing with setup servers on physical machines, client request is balanced within the different servers. These servers are characterized by their capacity which is different from one another and their load is determined by performing client tasks. Although the load balancing used on these servers may be efficient to balance the users' requests, the load provided by each physical node may not be efficient for a highly performance server utilization. Hence, load algorithm methods which use physical machine do not evenly partition the load to nodes. Therefore some machines get a big users' request depending on their capacity while other get small requests which flout the load balancing characteristics.

To overcome this shortage we introduced the concept of virtual servers in our farmer GIS servers for each computer. A virtual server is a completely isolated container capable of processing operation system and applications [18], behave like a physical machine and contains its own CPU, RAM, hard disk and interface card. It represents a node in the LHT in which routing, storage or retrieval of data items happen rather than at the physical node. Heterogeneity of node capacity is the main reason of using virtual servers per node than in the equal-capacity case, thus increasing the scalability of the system. Each virtual server node in the cluster is seeing itself as a physical device. A physical computer hosts one or more virtual servers depending of it capacity, it potential of failover, and load balancing is achieved by moving load from heavily to lightly VS nodes. When dividing physical machines into virtual servers, we must choose carefully the number of virtual servers to create since a huge 
number of virtual servers within a physical node may reduce the performance of the application system. One of the main repercussions of using virtual servers is the considerable reduction in the number of hardware computers used and the optimization of waste for capacity un-used on a single computer. Nevertheless the drawback of the utilization of these virtual servers increase the bandwidth and storage requirement [14] but this consideration is not great compared to the advantages.

\subsubsection{Load Balancer}

The system provides two load balancers, a primary load balancer which handles load balancing between the several GIS servers within the cluster and a secondary load balancer in case the primary load balancer failed. It leverages the servers' nodes to enhance responsiveness. Each VS node is configured to use the load balancers and will periodically send load and other information to the activated load balancer which will keep track of the load and availability of each VS node to which it is communicating (see LHT section below). Physical machines are also configured to use the load balancers and will allow the load balancer to get information about client request status whenever it is able to successfully perform the incoming request from the load balancer. The latter will keep track of the successful processing of each physical machine to which it is communicating in order to resend the same tasks to another physical node in case of failover. Since two load balancers are provided, a mechanism to activate the secondary engine is required in order the first fail. DNS load balancer is used to balance the two handlers which are not described in the document.

\subsubsection{Load Hash Table}

The LHT, like Distributed Hash Table in peer-to-peer system, manages a global identify [13]. The concept uses a unique identifier (global unique identifier (GUID)) which is associated with each virtual node in the cluster. The LHT maps the node ID to it corresponding RealTime-Weight-Load (RTWL), Real-Time-Jobs-Process
(RTJP) \& Real-Time-Successful-Process (RTSP). The RTWL is the real time load of a particular virtual server node. This is given by providing a web services script that calculates the virtual server process time at any minute and writing down into the loaded hash table. The RTJP is the real time set of jobs being processed by any particular node. The RTSP is the real time successful process provide by any node within the cluster. Within the LHT, we initialize the node with static values such as $\mathrm{C}_{\mathrm{I}}$, the capacity of the virtual server I (VSI); $\mathrm{U}_{\mathrm{L}}$, the Upper Loaded value that when a node receive that quantity of work it considered itself over-loaded; $\mathrm{L}_{\mathrm{L}}$, the Lower Loaded value that when a node receive that quantity of work it considered itself under-load. The LHT also provides the $\mathrm{VS}_{\mathrm{I}}$ Physical Node Membership (PNM) and Failover Likelihood $\left(\mathrm{F}_{\mathrm{L}}\right)$ of each virtual server to determine the probability of its failover which depends of the node's previous failover (Table 1).

LHT is implemented on the load balancers which are separated to physical machines. When the active load balancer gets a request, it firstly splits the incoming request into tasks (see request partitioning style in section 3.6 below), connect to the database, open and store tasks into the RST table and then open the LHT table and read the $F_{L}$ and either the capacity or RTWL of each nodes. After processing the algorithm in order to find out which server is more suitable to perform the task, the request is sent to the chosen node and the latter perform the task.

\subsubsection{Distributed Failover Callback Function Table (DFCFT)}

DFCFT is a table implements on each physical node (load balancer respectively) which allow the system to work out the virtual server failover (physical node respectively). It is introduced to help the chosen physical machine to resend the request to one of its virtual server when the former chosen VS fails. Since the connection between physical and virtual server nodes is an HTTP connection for rendering client request, the physical machines need a procedure to remind itself of the failing/

Table 1. Recapitulative table of the difference elements in the Loaded Hash Table (LHT).

\begin{tabular}{|c|c|c|c|c|c|c|c|c|}
\hline ID & Capacity & RTWL & RTJP & RTSP & FL & $\mathbf{U}_{\mathbf{L}}$ & $\mathbf{L}_{\mathbf{L}}$ & PNM \\
\hline ID. 1 & C. $1=18$ & 12 & $\mathrm{~J}_{2} \mathrm{~J}_{7}$ & - & $\underline{0.02}$ & 15 & 2 & PN.1 \\
\hline ID. 2 & C. $2=18$ & 9 & $\mathrm{~J}_{2}, \mathrm{~J}_{4} \mathrm{~J}_{6}$ & - & $\underline{0.01}$ & 15 & 2 & PN.3 \\
\hline \multirow[t]{4}{*}{ ID. 3} & C. $3=18$ & 10 & $\mathrm{~J}_{1}, \mathrm{~J}_{2} \mathrm{~J}_{7}$ & - & $\underline{0.01}$ & 15 & 2 & PN.1 \\
\hline & - & - & - & - & 0 & - & 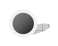 & - \\
\hline & - & - & - & - & - & - & 0 & - \\
\hline & - & - & - & - & - & 0 & 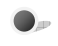 & 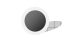 \\
\hline ID.N & C. $N=20$ & 13 & $\mathrm{~J}_{5}, \mathrm{~J}_{2}$ & & $\underline{0}$ & 16 & 2 & PN.4 \\
\hline
\end{tabular}


successful processes request; this is in order to send the same request to other virtual servers. Like LHT, the DFCFT uses web services implementation to inform the chosen PM that it's chosen virtual sever is either successful or not when processing the incoming request.

The table has the same concept as LHT in such a way that a unique identifier is associated to each virtual node within the physical node. DFCFT instantaneously maps the virtual server node ID to the incoming request and the client response status which suggests that the request has been successful or not. It contains the URL address of the request and a Boolean that indicates the request has failed or a response that indicates the request is successfully processed. A successful process of the request by the virtual server allows the physical node to get the result and then send it back to the load balancer for later reconstitution.

To implement the DFCFT we provide a relational database table (MSQL Server) within the physical node and access through web service by the virtual server node. The VS node after each successful or unsuccessful process sends a SOAP message to the web service to fill out the table. The physical node can then be aware of any virtual servers' failover.

The constitution of the table is given in the Table 2 and Table 3. Since nodes within any physical node can fail, a DFCFT is also implemented within the load balancer for PM Failover. Hence the load balancer can withdraw the request and then send it back to another physical machine depending on the algorithm until successful processing request or total GIS cluster failover.

\subsubsection{Request Split Table (RST)}

Since we proposed to split clients' request into tasks we need a table to store the parts for later reconstitution. For this to occur, we defined a request split table which store request identifier and request's tasks composition. RST is implemented within the load balancer. It allows the load balancer after the split of client requests into synchronous tasks, to store them in order to reconstitute the response of the original request. The composition of the RST is given in Table 4.

\subsection{Selecting Nodes and Routing Requests}

The Internet is comprised of many separate administrative domains or autonomous systems (ASs) [9] and routing request between them is entirely related to the request type and the software component. To route the client requests, the server side node needs to be selected in order to transit the request. We have to cases (Figure 1):

First incoming request: At the first time a client send request, several criteria can be use as parameters of choice. For example, the node with biggest capacity might be chosen. The choice among multiple suitable nodes might be randomized. If not randomized, the probability of distribution is, heavily based towards the best choice to ensure low route delay and/or strongly based on nodes that have the lowest failover likelihood. For instance, the first request or the first request with an empty pooling jobs (requests that come when there is no processing tasks within the cluster), the chosen physical server and virtual server nodes are made depending of

Table 2. Recapitulative table of difference elements in the DFCT-L1 for virtual server failover within the chosen physical machine.

\begin{tabular}{|c|c|c|}
\hline $\begin{array}{c}\text { Virtual } \\
\text { Server ID }\end{array}$ & Incoming Request & Status \\
\hline ID.1 & http://192.168.83.150:8080/3-dimentional-web-based-application/soa/monitoringservices/clip.jsp?cliptype=circle \&rnd= & False \\
\hline ID.2 & $\begin{array}{l}\text { http://192.168.83.10:8080/flood-web-based-application/soa/monitoringservices/mapinit.jsp?mapName=wh.Map\&rnd-0.5 } \\
948372403259459\end{array}$ & Result $_{2}$ \\
\hline ID.3 & http://192.168.5.90:8080/healthcarefacilities-web-based-application/soa/informationservices/findnearesthospital.jsp? & Result $_{3}$ \\
\hline ID.2 & $\begin{array}{c}\text { http://192.160.12.3:8080/3-dimentional-web-based-application/soa/routingservices/findpath.jsp?mapName=world.Map \& } \\
\text { rnd-0.5948372403259459 }\end{array}$ & False \\
\hline ID.4 & http://192.168.3.13:8080/flood-web-based-application/soa/locationservices/schoollocation.jsp? & False \\
\hline \multicolumn{3}{|c|}{$\begin{array}{l}\text { Table 3. Recapitulative table of difference elements in the DFCT }-\mathrm{L} 2 \text { for physical machine failover within the GIS serve } \\
\text { cluster. }\end{array}$} \\
\hline PM ID & Incoming Request & Status \\
\hline ID. 1 & http://192.168.83.150:8080/3-dimentional-web-based-application/soa/monitoringservice/clip.jsp? & False \\
\hline ID. 2 & http://192.168.83.10:8080/flood-web-based-application/soa/locationservics/mapinit.jsp? & Result $_{2}$ \\
\hline ID.1 & http://192.168.83.150:8080/3-dimentional-web-based-application/soa/monitoringservice/clip.jsp? & Result $_{1}$ \\
\hline
\end{tabular}


Table 4. Client Request Tasks Composition (Data partitioning request style: Rectangular Zoom In on a village).

\begin{tabular}{cccc}
\hline Client Tasks ID & Composition & \multicolumn{2}{c}{ Succession } \\
\hline$\bullet$ SOAP-Rect-ZoomIm-Request-X1Y1-X2Y2 & $\bullet$ Rect-Zoom-In & $\bullet$ Level $_{0}$ \\
$\bullet$ SOAP-Rect-ZoomIm-Request-X3Y3-X4Y4 & $\bullet$ Rect-Zoom-In & $\bullet$ Level $_{0}$ \\
$\bullet$ SOAP-Rect-ZoomIm-Request-X5Y5-X6Y6 & $\bullet$ Rect-Zoom-In & $\bullet$ Level $_{0}$ \\
$\bullet$ SOAP-Rect-ZoomIm-Request-X7Y7-X8Y8 & $\bullet$ Rect-Zoom-In & $\bullet$ Level $_{0}$ \\
\hline
\end{tabular}

the node's previous time in performing such request. The first chosen node in the cluster that satisfies the criteria is therefore picked. In case of failover, the second subsequent lightest virtual server node within the same physical node is chosen. In case of failover of the entire virtual servers of that chosen physical node, the next lightest physical node within the cluster is chosen. For our case, since there is no utilization value (first incoming request, utilization equal to zero), the Capacity (C) of the nodes (physical and virtual server nodes) and their Failover Likelihood (FL) are chosen as parameter of choice.

Subsequent requests: As nodes perform their associated tasks, the LHT is fulfilling gradually. To carry out the model we need to build a sub-script which reads each node capacity and process time (load) and then draw them in the corresponding address space in the LHT. This sub-script represents our mapping function, or hap map in the case of LHT. Thus the load balancer queries all nodes within the LHT to determine which one is more suitable for accepting the request depending of its weight-load. At first, the lightest physical node within the cluster is select to handle the client request depending of it utilization. And then a second query search is made to find out the lightest virtual server node within that specific physical node. If there are several nodes that satisfy to the lightest physical node criteria then the subsequent query search for the virtual server include the overall virtual servers to the satisfied PM.

The client requests in our GIS cluster are divided into two groups: simple requests, which do not require checking out the cache list in order to reduce the performing time and complex requests, which are stored on the cache memory. These request types are processed according to whether or not they have pre-process them

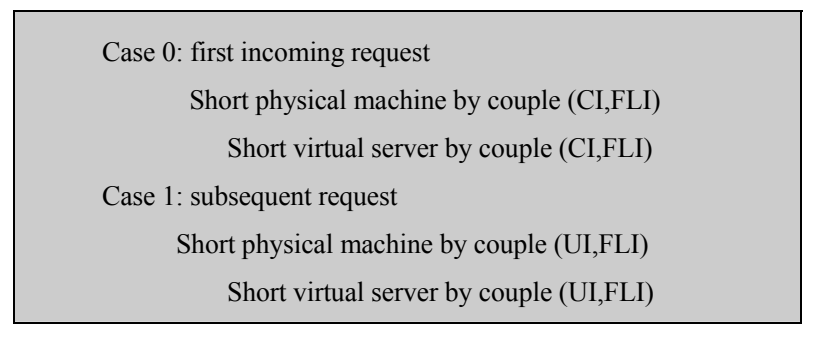

Figure 1. PM and VS shorting algorithm. before. Depending of the request type, the buffer cache list is sometimes checked to find out whether or not the request has a correspondence. As the node joins the GIS cluster, we just need to update the HLT table to make it operational. The algorithm is given in Figure 2.

\subsection{Nodes Failover (F)}

Nodes in peer-2-peer networks suffer from frequent transient failures $[11,19]$ and this scenario is also happening in distributed GIS. To handle failover node in distributed GIS, we defined the DFCFT table which contains request information and status that determines whether or not the client request is successfully processed. If the virtual server does not successfully process the request, the physical machine will pick up the request store into the DFCFT table and send it to another of its node. This method is repeated for all virtual servers within the physical machine until successful request or emptying virtual server within physical machines. Unsuccessful request is defined if a node is not responsive for a period time $\mathbf{L}_{\mathbf{T}}$ defined in the algorithm hence it is presumed failed. The weight-load is therefore equal to the default value of heavy-weight-load. The load balancer will no longer send message to the specific server until complete reparation. Moreover, every $\mathbf{T}_{\mathrm{F}}$ seconds, the algorithm (Figure 3) computes a schedule of virtual servers with the goal of detecting failure node with or without client request.

\subsection{Client Requests Partitioning (CRP)}

The proposed schema involves the subdivision of client's request into several tasks and each can be transferred to and processed by a different virtual server within the entire cluster nodes. The goal of this method is to considerably reduce the latency time for providing response. The method is to split client's request into smaller and separate tasks and transfer them to a lighter virtual server within a lightest physical machine node. It will reduce the latency time for performing request by processing pieces of the request at the same time. While this may improve the clients' response time, there is a risk of ex- 


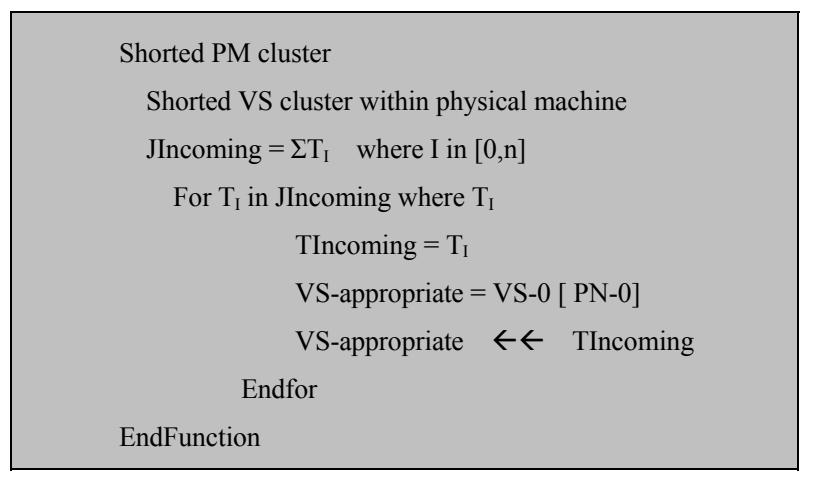

Figure 2. PM and VS selection algorithm.

LT is the latency time for performing such request

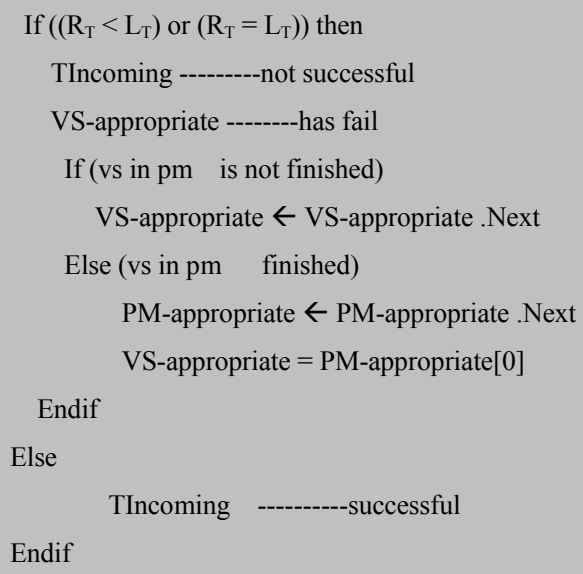

Figure 3. Load balancing system failover.

cessively fragmenting users' request. An increasing number of tasks would imply an augmentation in the overlay hop length and size of routing and processing. However the advantages provided by this method outweigh by far its disadvantages. Hence the number of fragments must be optimized depending of the request. However before dividing users' request into several tasks, we need to understand how request is being process within such systems.

Without any similar techniques, client sends request to the next tiers which are web server through the internet, GIS services through SAOP messages over HTTP, load balancing layer through HTTP protocol. In that latter layer, load balancer uses the algorithm and sends the request to a specific virtual server and after processing, the response is sent back to the client interface through the same path. Within the GIS virtual server, the client request constituted several functions processed subsequently, depending on the input values and computer memory space available. Even though there are set of functions that can be processed at the same time, the $\mathrm{CPU}$ processes the functions one by one, hence increase response time.

In case of splitting clients' request into synchronous and equal tasks, they can be processed at the same time in different GIS virtual servers and hence decrease response time leading to high responsiveness. We proposed to divide users' requests into two types in our D-GIS.

The first one is the request process as they are sending without any modification of the inputs. These requests are called the no-spitted requests. The second is the request that may split the input map into several pieces and process the task on each piece on different virtual GIS servers provided by the load balancing algorithm. For instance when a client request a zoom-in on a portion of the map, the latter can be divided into several equal maps and each part will be applied to the client request using a different virtual GIS server: Data Partitioning Request Style (see equation (A) in Figure 4). This method is mostly used to decompose simple GIS analysis functions. However some functions cannot be split using this method due to their implementation within the GIS servers. For instance the circle incoming map required a clip-by-circle function can not be divided due to the high-impossibility to decompose circle map into circular map pieces.

Case example: In the case of request that allow the client to select an interesting set of data for further information, the Data Partitioning Request Style is used on the incoming square of map. For that matter the request may be divided into four main tasks which are sending to four different GIS servers depending of the load balancing handler or manager. The client request is send to the web server which uses a software component to split the incoming map into four rectangular boundary maps. The four synchronous tasks are therefore performed by four different GIS servers and the result is sent back to the web server which replies to the request by displaying the four ranges of maps. By performing the synchronous tasks at the same time, the client response time is considerably reduced.

\subsection{Method Type Decomposition}

Before decomposing the incoming request, the system needs to identify the type of client request. Since we proposed to subdivided clients' requests into tasks and send them to different GIS servers, we need a technique to split request and store request's task composition and

$\mathbf{J}_{\text {incoming }}=\sum \mathbf{J}_{\mathbf{I}}$, where $1<\mathrm{I}<\{3,5,6\}$ depending

of the scale level (Local, national and international)

Figure 4. Equation A, data partitioning request style. 
later reconstitute the response to the divided request to send back to client interface. For implementing this method, several critical issues must be discussed.

How can the incoming request be split? Where can the client request (Client side or server side) be split? Which device is responsible of the split (load balancer or specific engine)? How can the client request be split to reconstitute meaningful processing tasks? How can the device responsible for request split recognize data partition request style and no-partitioning request? Does the client's incoming request contain all information needed to split the request? How can we have quasi-equal processing time in the different virtual server? To surrender for an efficient load balancing, we suggest that:

The load balancer is responsible for client's request split. A request can either be split or not. If a split is used, the request is a data partitioning request. Load balancer identifies data partition request style depending on the request input values. It can also identify, depending of the client request URL, the GIS functions the server need to call in order to process client's request.

The load balancer also contains a file that determines for each incoming client request the split method by identifying the method type of the latter request.

Depending of our split method, the overall input map is divided into twelve equal tasks. Each task from TI1 to TI12 has quasi-equal processing time. The following tables (Table 5 and Table 6) show the different splitting tasks. The first table, even though it brings better performance, may not be efficient due to the latency time for getting every response for reconstitution. If the split is not efficient we may be confront. However when splitting request into- quasi response time request, high performance can be achieve.

When working on the algorithm (Figure 5) we encounter several problems two of which are as follows.

Problems 1: To get sophisticated performance when balancing clients' request, we try to solve an important issue of load balancing which is persistent that is, where to store the information about the client requests so subsequent related-requests can be retrieve directly without performing the same process?

Problems 2: One critical issue must be discussing the balancing of client request into this cluster server. The issue is that each request that enters the system has an associate item which is composed of its popularity and the amount of processing time it needs to be served to a specific server or amount of serving time.

Problems 3: The decomposition of the request varies depending of the input area. Splitting for example the input query area into equal squares may not be efficient for performing tasks. Hence split is done depending of the quantity of information the input area contains: Quantity of Information (QoI).

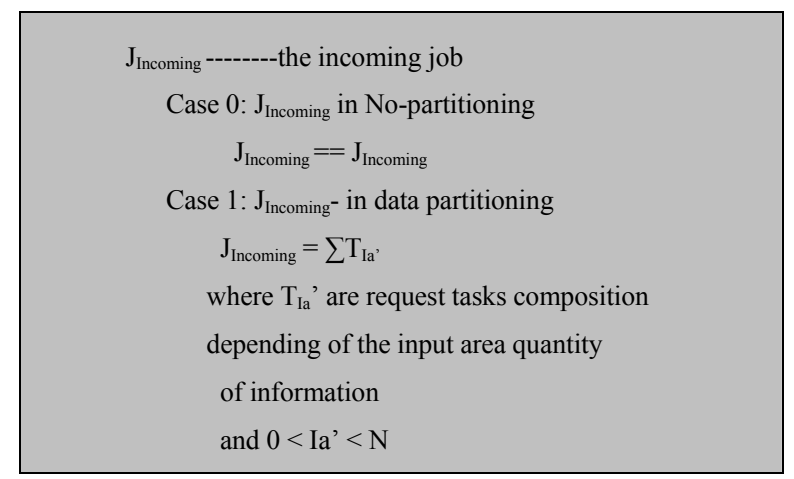

Figure 5. Client split request algorithm method.

Table 5. Table of the input area split map (equal range).

\begin{tabular}{ccc}
\hline $\mathbf{T}_{11}$ & $\mathbf{T}_{15}$ & $\mathbf{T}_{19}$ \\
$\mathbf{T}_{12}$ & $\mathbf{T}_{16}$ & $\mathbf{T}_{110}$ \\
$\mathbf{T}_{13}$ & $\mathbf{T}_{17}$ & $\mathbf{T}_{111}$ \\
$\mathbf{T}_{14}$ & $\mathbf{T}_{18}$ & $\mathbf{T}_{112}$ \\
\hline
\end{tabular}

Table 6. Table of the input area split map depending of the quantity of information.

\begin{tabular}{lcccc}
\hline$T_{111}$ & $T_{11}$ & $T_{12}$ & $T_{13}$ & $T_{18}$ \\
\hline \multirow{2}{*}{$T_{110}$} & $T_{15}$ & $T_{112}$ & $T_{17}$ \\
& & $T_{16}$ & $T_{14}$ & $T_{11}$ \\
\hline
\end{tabular}

\subsection{Dynamic Distributed Load Balancing Architecture}

Our Dynamic Distributed Load Balancing architecture is composed essentially of four elements: Load Balancers, Load Algorithm, DFCFT and a LHT. The Load Balancer which is a single server between the GIS servers cluster and the GIS services cluster receives the client request from the web server. Our design proposed the use of two load balancers in case the first load balancer fails. The secondary load balancer is configured to take over the client request balancing to provide high availability of the system. Load Algorithm control the selecting of physical and virtual server nodes, write information onto the LHT and DFCFT. The DFCFT controls the system failover by sending back the request to the physical node in order for the latter to redirect it to another virtual server. At last we have, the LHT, controlled by the load algorithm. The distributed dynamic load balancing architecture is given in the following figure (Figure 6).

\subsection{Dynamic Distributed Load Balancing Algorithm}

Since it is very hard to address the load balancing prob- 


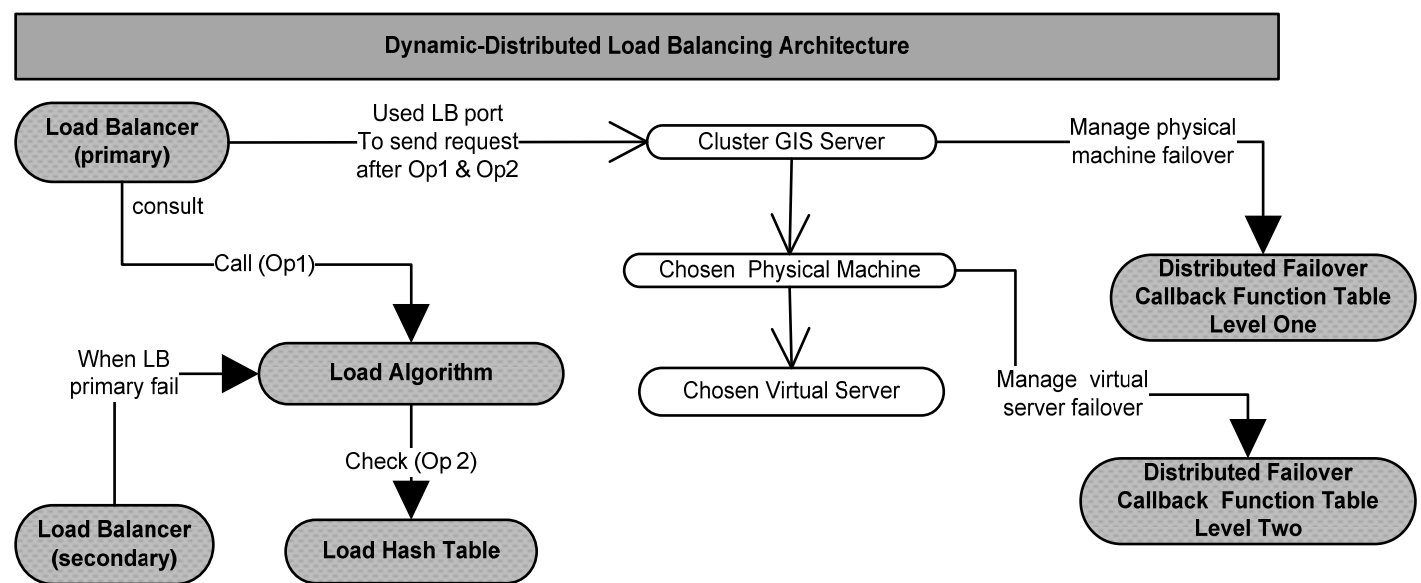

Figure 6. Dynamic \& distributed load balancing general architecture in distributed GIS.

Table 7. Definition of some parameters formula.

\begin{tabular}{|c|c|c|}
\hline $\mathrm{U}_{\mathrm{i}}=\mathrm{W}_{\mathrm{i}} / \mathrm{C}_{\mathrm{i}}$ & Mac & $\mathrm{I}=\sum \mathrm{VS}_{\text {Ia }}$ where $1<\mathrm{a}<\mathrm{n}$ \\
\hline$R T S P=\left\{\begin{array}{cl}\mathrm{W}_{\mathrm{i}} & \text { if } \mathrm{W}_{\mathrm{i}}<\mathrm{C}_{\mathrm{i}} \\
\mathrm{C}_{\mathrm{i}} & \text { if } \mathrm{W}_{\mathrm{i}}>\mathrm{C}_{\mathrm{i}} \\
0 & \text { if node fail }\end{array}\right.$ & $\begin{array}{c}\mathrm{W}_{\mathrm{i}}=\sum \mathrm{Wvs}^{\mathrm{n}} \\
\text { where vs №1 }<\mathrm{vs}^{\mathrm{n}}<\text { vs №n }\end{array}$ & $\begin{array}{l}\mathrm{FL}=\mathrm{RTSP} / \mathrm{N}_{\text {JobsAssigned }} \\
\text { where } \mathrm{N}_{\text {JobsAssigned }} \text { is the number of jobs send to node }\end{array}$ \\
\hline
\end{tabular}

lem in its full generality, we make some simplifying assumptions, which we believe are reasonable in practice for a distributed-based GIS application. Some symbologies used are also presented in Table 7.

- A job $\mathrm{J}$ is a task in the split request table and must run contiguously on one machine.

- Arrived job J sent to node I may be processed by the node itself

- It may be transferred to another node if there is failover.

- Each node I has N virtual servers VSI named VSI1, VSI2, VSI3...SIn which we limit to 3.

- The $C_{i}$ is the capacity of the physical node I and $\mathrm{CVS}_{\mathrm{ia}}$ is it virtual server a, which is related to for example, available disk space, processor speed, or bandwidth.

- A node's utilization $U_{i}$ is the fraction of its capacity $\mathrm{C}_{\mathrm{i}}$ and it load $\mathrm{W}_{\mathrm{i}}$ [8] and [12].

- Finally each virtual server can process at least one job at a time.

We consider that the associate item is equal for each incoming request. Then they are negligible for the purpose of this study.

We decided for the purpose of the model to keep client information in the web server layer for an efficient load balancing.

\section{DDLB Prototype Design}

D-GIS is defined as the use of internet technologies to distribute geographic information in a variety of forms, including maps, images, datasets, spatial analysis operations and reports. It is an advanced model which combines both an advancement of internet GIS and Mobile GIS advanced models. The distributed GIS encounter the most complete GIS system and embed Mobile GISs and Web-GIS. Its architecture is therefore as general as possible. The next figure will present the prototype design proposed in this paper. This design will focus of the system layering model and particularly of the two load balancing layers (Figure 8).

By incorporating the Network Load Balancing and the Dynamic Distributed Load Balancing model into our distributed GIS, we obtain the overall system schema which provides high performance and responsiveness distributed GIS application. The schema is composed of five system tiers (client tier, web server tier, GIS web services tiers, GIS servers tier and spatial data servers tier) and two algorithm layer models.

\section{Result and Discussion}

For assuring the effectiveness of our method, we provide a simulation that shows satisfaction. Although the proposed algorithm is quiet long (Figure 7), it simplicity and speed allows the load balancing to deliver very high performance, including both high throughput and low response time, in a wide range of useful GIS applications. For example when one web-based GIS client requests for a 
Step 0: update LHT nodes after assignation

Step 1: Select the load balancer use to dispatch client's requests into cluster servers

If (LBPrimary working)

Step 2: Loop all Jobs send to the load balancer handler

Step 3: split the incoming request into synchronous and asynchronous tasks

Step 4: Open the LHT and get the information about each virtual server

Step 5: call the shorting function to range PM and its VS from lightest to heaviest

Step 6: Select appropriated VS of appropriate PM and assign synchronous tasks

Step 7: task being process by the chosen virtual server within the chosen PM

Step 8: call the failover function to find out whether task fails or not

Step 9: select the next incoming job

Figure 7. Dynsmic distributed load balancing algorithm.

Table 8. MapGIS K9 server-based response time before and after load balancing (millisecond).

\begin{tabular}{|c|c|c|}
\hline Characteristics & Map Request Response Time & MapGIS-K9 IMS \\
\hline \multirow[t]{2}{*}{ No web caching } & Time per Map at launch time & 54000 \\
\hline & Time per map to access for displaying vector map & 5500 \\
\hline \multirow[t]{3}{*}{1 client on 1 GIS server } & Time per map to access for displaying tile map & 600 \\
\hline & Time per map to individual access for query & 4300 \\
\hline & Average time for simultaneous displaying & 16300 \\
\hline \multirow[t]{3}{*}{4 clients on 1 GIS server } & Time per map to access for displaying tile map & 945 \\
\hline & Average time for simultaneous query & 12690 \\
\hline & Average time for simultaneous displaying vector & 4530 \\
\hline \multirow[t]{3}{*}{45 clients on 4 GIS server } & Average time for simultaneous displaying tile map & 1165 \\
\hline & Average time for simultaneous query & 4396 \\
\hline & Average time for simultaneous displaying vector & 3482 \\
\hline \multirow[t]{2}{*}{$\begin{array}{l}45 \text { clients on } 5 \text { virtual } \\
\text { GIS servers }\end{array}$} & Average time for simultaneous displaying tile map & 963 \\
\hline & Average time for simultaneous query & 3836 \\
\hline
\end{tabular}

query operation, the web server receives spatial query request from loading the web page through the client web browser. The load balancer receives the spatial query from the web server, partitions the input region into uniform tile-based regions using spatial operation, assigns the partitioned regions to several GIS servers, allows one GIS server to process queries for a certain region, and dynamically reallocate GIS servers for processing the queries by receiving each GIS server query processing response of it regions.

By calling map initialization, query processing, select regions functions into the distributed application, the client response time is highly reduced. The GIS server setups on virtual servers are therefore preventing the concentration of work on the system.

We show that unlike previous methods of providing high performance and responsiveness in distributed GIS, our model is tied to the number of virtual servers used, their capacity and frequency of failover and consequently provide high performance load balancing. Yet, the model can handle the heterogeneity of a distributed GIS system, system failover of virtual servers and physical machines, and high scalability to meet future need. We can see in Table 8 that the response time for an individual query task before applying solution (4300) are almost the same as the response time for simultaneous query task on a cluster GIS server (4396).

\section{Conclusions and Future Works}

The aim of the work was to provide solution for high performance and responsiveness distributed GIS 


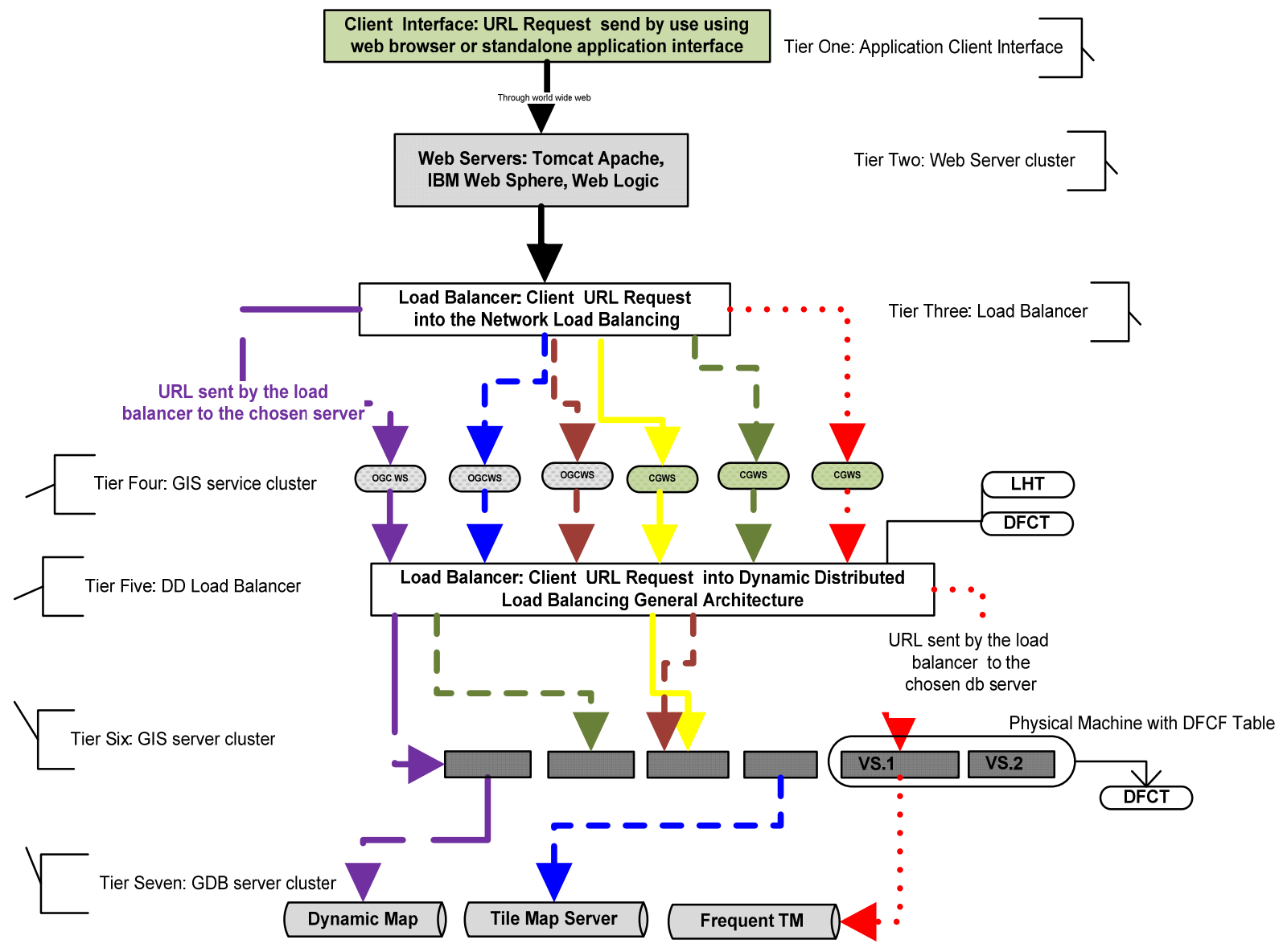

Figure 8. Dynamic \& distributed-based load balancing general configuration in distributed GIS system.

in order to overcome distributed GIS problems which are encountered on the rapidity of distributed GIS applications. Several methods and techniques have been introduced for that matter but few researches were focused on load balancing in GIS system. By incorporating a Dynamic Distributed load balancing model into our distributed GIS, we obtain the overall schema which enhances GIS application effectiveness.

The performance carries out the action or accomplishes of task, especially the one requiring care or skill [9]. It is defined as the possibility of optimizing, which is the enhancement of effectiveness and is related directly to the execution time of the computers in which the task is being processed and depended on the interfaces between the processor and the memory.

In D-GIS, the performance of application is an overwhelming issue since rendering the combination of huge data and information, multiple simultaneous clients' requests and cross processes and platforms call from client to server brings down the latency time to provide responses. Our solution provides high scalability, high availability, and good load balancing capabilities; hence GIS system organization can increase their business force by making clients happy to use their software.

\section{Acknowledgements}

The work is supported by National "863" plan: Research and software development of three-dimensional spatial information service technique of the network (No.2009AA12Z211).

\section{References}

[1] S. Malik, "Dynamic Load Balancing in a Network of Workstation," 95.515 Research Report, 19 November, 2000 .

[2] Sandeep Sharma, Sarabjit Singh, and Meenakshi Sharma, "Performance Analysis of Load Balancing Algorithms," Proceedings of World Academy of Science, Engineering and Technology, Vol. 38, 2008.

[3] Y. R. Lan, "A Dynamic Load Balancing Mechanism for Distributed Systems," Journal of Computer Science and Technology, Vol. 11, No. 3, 1996, pp. 192-207.

[4] A. Sayer, "Thesis Proposal: High Performance, Federation and Service-Oriented Geographic Information Sys- 
tems," Indiana university, Indiana.

[5] P. S. Zhao and C. J. Yang, "The Study on the Architecture of Web-GIS by Institute of RS Applications," Chinese Academy of Sciences,Beijing.

[6] D. L. Eager, E. D. Lazowska and John Zahorjan, "Adaptive Load Sharing in Homogeneous Distributed Systems," IEEE Transactions on Software Engineering, Vol. 12, No. 5, May 1986, pp. 662-675.

[7] A. Sharma, S. Singh, and Meenakshi, "Sharma: Performance Analysis of Load Balancing Algorithms," Proceedings of World Academy of Science, Engineering and Technology, Vol. 38, 2008.

http://masters.donntu.edu.ua/2010/fknt/babkin/library/article11.pdf

[8] B. Godfrey, K. Lakshminarayanan, S. Surana, R. Karp and I. Stoica, "Load Balancing in Dynamic Structured P2P Systems," Performance Evaluation, Vol. 63, No. 3, 2006, pp. 217-240. http://www.ieee-infocom.org/2004/Papers/46_4.PDF

[9] J. Feigenbaum and S. Shenker, "Distributed Algorithmic Mechanism Design Recent Results and Future Directions," DIALM '02 Proceedings of the 6th international workshop on Discrete algorithms and methods for mobile computing and communications, New York, 2002.

[10] T. Horanont, N. K. Tripathi and R. Raghavan, "A Comparative Asseessment of Internet GIS Server systems," Space Technology Applications and Research Program, School of Advanced Technologies Asian Institute of Technology, Thailand, 2005.

[11] W. J. Bolosky, J. R. Douceur, D. Ely and M. Theimer, "Feasibility of a Serverless Distributed File System an Existing Set of Desktop PCs," Proceedings of the deployed on International Conference on Measurement and Modeling of Computer Systems, Santa Clara, CA, June 2000, pp. 34-43.

[12] J. Ledlie and M. Seltzer, "Distributed, Secure Load Bal- ancing with Skew, Heterogeneity, and Churn," Division of Engineering and Applied Science, Harvard University, September 2009.

http://dash.harvard.edu/bitstream/handle/1/2962660/Seltz er_DistributedSecure.pdf? sequence $=2$

[13] P. B. Godfrey and I. Stoica , "Heterogeneity and Load Balance in Distributed Hash Tables," Computer Science Division, University of California, Berkeley.

[14] D. R. Karger and M. Ruhl, "Simple Efficient Load Balancing Algorithm for Peer-to-Peer Systems," SPAA '04 Proceedings of the sixteenth annual ACM symposium on Parallelism in algorithms and architectures, 2004. http://static.googleusercontent.com/external_content/untrusted_dlcp/research.google.com/fr//pubs/archive/33339. pdf

[15] A. Rao, K. Lakshminarayanan, S. Surana, R. Karp and I. Stoica, "Load Balancing in Structured P2P Systems," Proceedings of IEEE INFOCOM, February, 2003. http://www.ieee-infocom.org/2004/Papers/46_4.PDF

[16] F. Dabek, M. F. Kaashoek, D. Karger, R. Morris and I. Stoica, "Wide-area Cooperative Storage with CFS," SOSP '01 Proceedings of the eighteenth ACM symposium on Operating systems principles, Vol. 35, No. 5, pp. 202-215.

[17] Hash Table from Wikipedia the Free Encyclopaedia, Access on April 2010. http://en.wikipedia.org/wiki/Hash_table

[18] Virtual Server form Wikipedia. http://en.wikipedia.org/wiki/Virtual_private_server

[19] L. P. Cox and B. D. Noble, "Samsara: Honor among Thieves in Peer-to-Peer Storage," Department of Electrical Engineering and Computer Science, University of Michigan Ann Arbor, MI 48109-2122. Page 4. http://www.eecs.harvard.edu/ mema/courses/cs264/paper s/samsara-sosp2003.pdf 